Revisión

\title{
Papel de la radioterapia en el tratamiento del carcinoma in situ de mama
}

\author{
F. J. Andreu Martínez, J. M. Martínez Mateu
}

\section{Resumen}

Como consecuencia de los programas de cribado o despistaje mamográficos, la incidencia de tumores de mama no invasivos ha aumentado de forma sorprendente. Sin embargo, cual debe ser el tratamiento de elección para este tipo de tumores no ha sido bien establecido y ha dejado la controversia de la mejor opción terapéutica a realizar, la mastectomía o el tratamiento conservador. Se ha realizado una revisión de la literatura, así como, una discusión de las alternativas de tratamiento posibles en el abordaje terapéutico del carcinoma in situ de mama, en especial del papel de la radioterapia.

\section{Palabras clave:}

Cáncer de Mama. Radioterapia. Cirugía. Hormonoterapia.

Oncología, 2006; 29 (8):338-344 


\section{Summary}

As a result of mammographic screening programs, the incidence of diagnosed non-invasive breast carcinomas has increased markedly. Nevertheless, the elective treatment for these kind of tumours has not yet been established, leading to controversies to decide about which is the best therapeutic option, whether mastectomy or conserving therapy. We make a review of the literature, and discuss the different treatment options for breast carcinoma in situ, especially the role of radiation therapy.

Key words: Breast neoplasms. Carcinoma in situ. Radiotherapy. Surgery. Hornone treatment.

\section{Introducción}

Los carcinomas no invasivos de mama incluyen la enfermedad de Paget del pezón, y dos entidades histopatológicas que son diferentes tanto en su presentación clínica como en su comportamiento biológico: carcinoma lobulillar in situ (CLIS) y el carcinoma ductal in situ (CDIS). Como consecuencia de los programas de cribado o despistaje mamográficos, la incidencia de este tipo de tumores ha aumentado de forma sorprendente. No obstante el tratamiento de elección para el cáncer no invasivo de mama, todavía no ha sido bien establecido.

\section{Carcinoma lobulillar}

El CLIS ha de considerarse como un factor de riesgo para desarrollar un carcinoma infiltrante de mama, que habitualmente es de tipo histológico ductal. Este riesgo de padecer un carcinoma infiltrante es según los estudios entre un 10-35\% a los 20-25 años y afecta casi por igual a ambas mamas, esto es, un riesgo entre 9 y 12 veces mayor que el de la población general ${ }^{1}$.

La recomendación de tratamiento más ampliamente aceptada en la actualidad es la exéresis de la lesión y el seguimiento de la paciente. Sin embargo, la mastectomía subcutánea bilateral más reconstrucción inmediata podría tener indicaciones en pacientes con historia familiar de cáncer de mama invasivo o con una conocida predisposición genética ${ }^{2}$.

La radioterapia (RT) no juega ningún papel en el tratamiento del CLIS.

La utilización de tamoxifeno en la prevención del cáncer de mama en pacientes diagnosticadas de CLIS puede ser una buena opción a la observación exclusiva, ya que, administrado durante 5 años, ha demostrado disminuir la incidencia de otros tumores de mama en un $56 \%{ }^{3}$.

\section{Enfermedad de Paget}

Esta entidad se caracteriza histológicamente por la presencia de células de Paget localizadas por toda la epidermis ${ }^{4}$. La presentación clínica de la enfermedad de Paget es la de, en apariencia, una erupción benigna eczematosa crónica del complejo areola pezón que se cura y recidiva de forma repetitiva. Las secreciones por el pezón, el prurito, y el dolor están frecuentemente asociadas, y la lesión puede progresar hasta producirse erosión, retracción del pezón y sangrado. En los casos muy avanzados puede verse afectación de la piel periareolar.

Casi en un $95 \%$ de los casos, la enfermedad de Paget está asociada con un tumor maligno de mama que puede ser palpable o no palpable. De todos los casos, el 50\% está asociado con una masa palpable y de estos el 90-94\% es un cáncer invasivo de mama. Cuando no hay una masa palpable, el CDIS es el tumor asociado en un 66-86\% 5 .

El tratamiento varía en función de si el tumor es infiltrante o no. La mastectomía ha sido considerada como el tratamiento de elección; sin embargo más recientemente se está estudiando la posibilidad de realizar un tratamiento conservador, escisión local exclusiva, RT exclusiva o combinación de ambas ${ }^{6}$. Bijker y colaboradores han publicado en un estudio multicéntrico auspiciado por la European Organiza- 
tion for Research and Treatment Cancer (EORTC) Study 10873, que en 61 pacientes diagnosticadas de enfermedad de Paget sin padecer un cáncer infiltrante y sometidas a un tratamiento conservador consistente en una escisión local o del complejo areola pezón, hasta conseguir márgenes negativos, y RT al volumen mamario restante y con una mediana de seguimiento de 6,4 años un porcentaje de recidiva local a los 5 años del $5,2 \%{ }^{7}$.

\section{Carcinoma ductal}

El tratamiento de las pacientes con CDIS de la mama esta siendo en la actualidad un punto muy controvertido, además tras la implantación de los programas de cribado o despistaje supone alrededor del 20\% de los cánceres de mama diagnosticados por mamografía. El CDIS puede presentarse como nódulo palpable o como una lesión no palpable detectada por mamografía, generalmente en forma de microcalcificaciones, siendo esta forma la presentación clínica más frecuente en la actualidad.

Bajo el térmico CDIS se agrupa a un conjunto de lesiones, que de los estudios realizados conocemos que difieren, en su presentación clínica, características mamográficas, extensión y distribución dentro de la mama, rasgos histológicos y marcadores biológicos. Además del seguimiento realizado tenemos información de que estas lesiones también varían en su patrón de recidiva o progresión hacia un cáncer infiltrante de mama.

Han sido identificados algunos marcadores moleculares asociados con la aparición de tumores de mama. El receptor estrogénico, esta expresado en el $70 \%$ aproximadamente de los CDIS. El HER2/neu, un proto-oncogen, está sobreexpresado en casi la mitad de los CDIS, pero no en la hiperplasia atípica ${ }^{8}$. El p53, un tumor-gen supresor, está mutado en aproximadamente un $25 \%$ de los CDIS, pero es muy raro encontrarlo mutado en proliferaciones benignas de mama ${ }^{9}$. Por tanto, la frecuencia con la que estos marcadores moleculares estén expresados en un CDIS, nos orientará hacia su posible transformación en un cáncer invasivo.

La mastectomía ha sido el tratamiento de elección del CDIS, siendo un tratamiento muy eficaz con un porcentaje de control loco-regional entre el $96 \%$ y el $100 \%$, y con una mortalidad específica por cáncer inferior al $4 \%{ }^{10}$. No existen estudios rando- mizados que comparen la mastectomía y el tratamiento conservador de mama como mejor opción terapéutica del CDIS.

Del tratamiento conservador de mama del CDIS, existen dos estudios randomizados que comparan la escisión local frente a escisión local más RT. En el NSABP B-17, el porcentaje de recidiva local a los 12 años fue del 15,7\% para el grupo que llevó RT frente al 31,7\% que no la llevó (p <0.000005). En el ensayo de la EORTC 10853, el porcentaje de recidiva local a los 4 años fue del $9 \%$ para el grupo que llevo RT, comparado con el $16 \%$ del grupo que no fue tratado con RT $(p=0,005)^{11,12}$. No obstante, en ninguno de los dos estudios existe una ventaja en términos de supervivencia. También se les critica que ninguno de los dos trabajos logre identificar subgrupos donde la RT pueda ser omitida. Los estudios retrospectivos sugieren que la RT puede no ser necesaria tras un escisión local con márgenes amplios ( $\geq 10 \mathrm{~mm}$ ), grado histológico y/o nuclear bajo o subtipo no comedocarcinoma ${ }^{13}$.

En cuanto al papel del tamoxifeno en el tratamiento del CDIS, existe un ensayo clínico randomizado, el NSABP B-24, que compara escisión local más RT, con escisión local más RT y tamoxifeno. Las pacientes que recibieron tamoxifeno presentaron un disminución en la incidencia de acontecimientos adversos relacionados con cáncer de mama $(8,2 \%$ frente al $13,4 \%$ a los 5 años, respectivamente, $\mathrm{p}=0,0009)$, sin embargo no demostró una mejoría en la supervivencia ${ }^{14}$. Por contra, en el otro ensayo clínico randomizado, llevado a cabo por el UKCCR, Ductal Carcinoma in situ Working Party y el DCIS trialists in the UK, Australia y New Zealand, valorando el papel del tamoxifeno y RT, aunque existe beneficio, no es estadísticamente significativo $^{15}$. El papel del tamoxifeno en ausencia de RT sobre la mama no ha sido todavía establecido. En este sentido está en marcha un ensayo clínico auspiciado por el Radiation Therapy Oncology Group (RTOG, protocolo 9804), que intenta responder a esta incógnita.

De la revisión de la literatura parece existir una serie de factores adversos que condicionan un incremento de las recidivas locales. Por este motivo en 1996 se introdujo el índice pronóstico de Van Nuys (VNPI), inicialmente se establecieron tres factores independientes de recidiva local (tamaño tumoral, grado histológico y estado de los márgenes quirúrgi- 
TABLA I

Factores pronósticos del DCIS, según el índice USC/VNPI y puntuación alcanzada (valores entre 1-3), según los parámetros o características descritas.

\begin{tabular}{|lccc|}
\hline Índice USC/VNPI (factores pronósticos) & & Graduación & \\
& 1 & 2 & 3 \\
\hline Clasificación patológica & Bajo grado sin necrosis & Bajo grado con necrosis & Alto grado \\
Márgenes quirúrgicos & $>10 \mathrm{~mm}$ & $1-10 \mathrm{~mm}$ & $<1 \mathrm{~mm}$ \\
Tamaño tumoral & $\leq 15 \mathrm{~mm}$ & $16-40 \mathrm{~mm}$ & $\geq 40 \mathrm{~mm}$ \\
Edad & $\geq 60$ años & $40-59$ años & $<40$ años \\
\hline
\end{tabular}

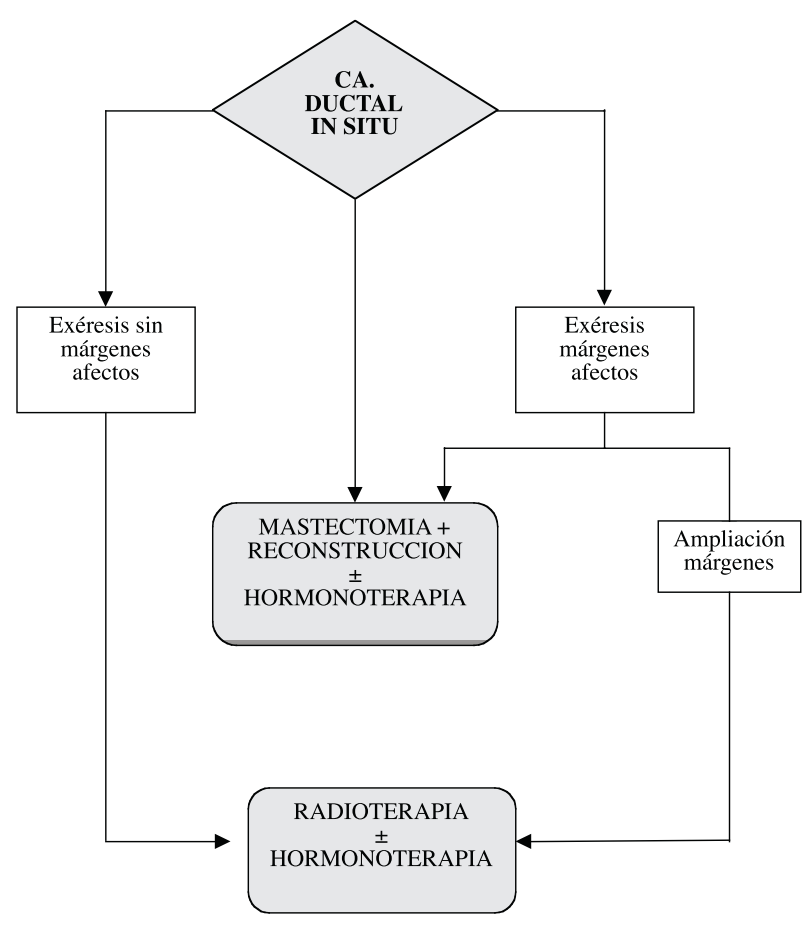

Figura 1. Arbol de decisión del tratamiento del carcinoma ductal in situ (DCIS).

$\cos )^{16}$, posteriormente este índice se amplió hasta cuatro, (presencia o ausencia de comedoconecrosis) o cinco factores (edad), y pasó a denominarse University of Southern California/Van Nuys Prognostic Index (USC/VNPI) ${ }^{17,18}$. Sin embargo, parece que los indicadores de riesgo de recidiva local son fundamentalmente la edad inferior a 40 años y márgenes quirúrgicos libres de resección $<10 \mathrm{~mm}^{19,20}$. No obstante, son necesarios estudios prospectivos bien diseñados para confirmar estos puntos, ya que mucha de la evidencia existente proviene de estudios retrospectivos o estudios prospectivos que entre sus objetivos no estaba el valorarlos ${ }^{21,22}$.

La estrategia terapéutica para las pacientes podría individualizarse y ser establecida según los cuatro criterios pronósticos propuestos en el índice USC/VNPI, a cada uno de ellos se le asigna una puntuación que oscila entre 1 y 3 puntos, en función de determinadas características (Tabla I). Si se obtiene una puntuación entre 4 y 6 , el tratamiento de elección es el quirúrgico conservador, sin RT postoperatoria; si se alcanza una puntuación entre 7 y 9 el tratamiento de elección es el quirúrgico conservador, con RT postoperatoria, finalmente si se alcanza una puntuación entre 10 y 12 el tratamiento de elección es la mastectomía (Figura 1).

La realización de una linfadenectomía axilar "clásica" no esta indicada para el CDIS, no obstante, el papel que puede aportar la biopsia del ganglio centinela, está por determinar en casos seleccionados, es decir en pacientes con factores de alto riesgo asocia$\operatorname{dos}^{23}$.

\section{Volúmenes a irradiar}

El volumen aceptado como estándar es la glándula mamaria en su totalidad. No obstante, se demuestra en algunos ensayos, que la gran mayoría de las recidivas se presentan a nivel del lecho tumoral, y no en otros cuadrantes mamarios, como lo cuantifica el estudio de Milán ${ }^{24}$ en el que el $85 \%$ de las recidivas intra-mamarias se produce en la región de la cicatriz y sólo el $15 \%$ en otros cuadrantes, porcentaje este último similar al de recidivas detectadas en la mama contralateral. Podría plantearse por tanto, que si para el carcinoma invasivo puede bastar la irradiación del lecho tumoral o cuadrante afecto en determinadas pacientes de bajo riesgo de recaída 
(edad >60 años, estadios T1N0, RE positivos...), también sería posible este planteamiento para los carcinomas no invasivos. De todos modos debe esperarse a los resultados de otros ensayos en curso para adoptar una decisión definitiva, no recomendándose su práctica fuera de ellos. Se trata de ensayos que comparan la irradiación tradicional de toda la glándula con la RT de un solo cuadrante, bien externa conformada $3 \mathrm{D}^{25,26}$, bien braquiterapia ${ }^{27,28}$, bien RT intraoperatoria ${ }^{29}$.

\section{Dosis y fraccionamiento}

La dosis que debe administrarse en la glándula mamaria tras la cirugía conservadora, aceptada como estándar, es de 46-50 Gy, con un ritmo de 180200 cGy por sesión, 5 veces por semana. Sólo en caso de márgenes afectados y si se rechaza la reescisión para ampliar éstos, estaría indicada una sobredosis limitada al lecho tumoral ("boost"), sobredosis que puede administrarse tanto con RT externa, habitualmente electrones, como con braquiterapia ${ }^{30,31}$.

\section{Técnica de irradiación de la glándula mamaria}

La técnica que puede considerarse estándar es la de dos campos de fotones, tangenciales a la pared costal, con energías de 4-6 MV, partiendo siempre de una planificación 3D tras simulación por medio de TAC. Puede aceptarse la utilización de haces de Co60 si el volumen mamario no es importante. Se recomienda la introducción de segmentos adicionales que cubran los volúmenes mamarios subdosificados para mejorar la homogeneidad de dosis, lo que es especialmente necesario en mamas voluminosas ${ }^{32}$.

\section{Plazos de administracion de la radioterapia}

Hay evidencia científica que sostiene, desde el punto de vista biológico, que el retrasar el inicio de radioterapia tiene un efecto negativo en los resultados del tratamiento ${ }^{33}$. Existen datos clínicos en el caso de los tumores de cabeza y cuello, así como en el cáncer de cérvix ${ }^{34}$. Sin embargo en el caso de pacientes con cáncer de mama con o sin indicación de recibir quimioterapia, la relación entre el riesgo de incremento de recidivas locales y el intervalo entre la cirugía y el inicio de la radioterapia es muy controvertido.
A este respecto, existen dos revisiones sistemáticas. Huang et al. ${ }^{35}$ concluye que el porcentaje de recidivas locales a los 5 años fue significativamente mayor en el grupo de pacientes tratadas con radioterapia adyuvante por cáncer de mama transcurridas más de 8 semanas tras la cirugía, que aquellas en que la irradiación se efectuó antes de las 8 semanas. Hebert-Croteau et al. ${ }^{36}$, que efectúan una revisión crítica de los mismos artículos utilizados por Huang, concluye que la radioterapia es efectiva en controlar la enfermedad residual microscópica independientemente del momento en que se inicie el tratamiento ionizante y que en el caso concreto del cáncer de mama, no existe una evidencia firme que sostenga que el retraso en el inicio de la radioterapia conlleva un efecto adverso en los resultados del tratamiento $^{37}$. Esta discrepancia ha llevado a otros autores a sugerir que otros factores podrían modular el impacto del intervalo de tiempo entre cirugía y RT (estado de los márgenes?) ${ }^{38}$.

Sin embargo, un documento de consenso canadiense, recomienda que todas aquellas pacientes que hayan sido sometidas a un tratamiento quirúrgico conservador por cáncer de mama, deberían iniciar la radioterapia no más tarde de 12 semanas tras la cirugía ${ }^{39}$. También en el Reino Unido recomiendan que el intervalo de tiempo entre la cirugía conservadora de cáncer de mama y el inicio de la radioterapia no sea superior a 4 semanas (no exceder de los 20 días laborables) ${ }^{40}$. No obstante, parece que en pacientes diagnosticadas de cáncer de mama en estadios I y II, el efecto que tiene el retrasar el inicio de la radioterapia es pequeño, y puede suponer un incremento absoluto en el porcentaje de recidivas locales de $1 \%$ por mes de retraso ${ }^{41}$, o aunque pueda comprometer el control local no afecta a la supervivencia $^{42}$. En el caso de los CDIS, se podrá iniciar la radioterapia, a partir de los 15 días de la cirugía, y se recomienda hacerlo antes de 2 meses.

\section{Contraindicaciones de la radioterapia}

Dentro de las contraindicaciones para administrar RT en pacientes diagnosticadas de cáncer de mama se consideran dos grupos:

- Contraindicación absoluta: el embarazo.

- Contraindicación relativa: las enfermedades autoinmunes o del colágeno, así como la anatomía inadecuada de la mama ${ }^{43,44}$. 


\section{Bibliografía}

1. Ottesen GL, Graversen HP, Blichert-Toft M, et al. Carcinoma in situ of the female breast: 10 year follow-up results of a prospective nationwide study. Breast Cancer Res Treat 2000; 62: 197-210.

2. Fisher ER, Costantino JP, Fisher B, et al. Pathologic finding from the National Surgical Adyuvant Breast Project (NSABP) Protocol B-17: five-years observations concerning lobular carcionoma in situ. Cancer 1996; 78: 14031416.

3. Fisher ER, Costantino JP, Wickerham DL, et al. Tamoxifen for prevention of breast cancer: report of the National Surgical Adyuvant Breast and Bowel Project P-1 Study. J Natl Cancer Inst 1998; 90: 1371-1388.

4. Jacobs TW, Ricci A, Deckers PJ. Paget's disease of the nipple-areola complex. Surg Clin North Am. 1996; 76: 365-381.

5. Sakorafas GH, Blanchard K, Sarr MG, et al. Paget`s disease of the breast. Cancer Treatment Reviews. 2001; 27: 9 18.

6. Pierce LJ, Haffty BG, Solin LJ, et al. The consevative managament of Paget's disease of the breast with radiotherapy. Cancer 1997; 80: 1065-1072.

7. Bijker N, Rutgers EJT, Duchateau L, et al. Breast-conseving therapy for Paget's disease of the nipple. Cancer 2001; 91: 472-477.

8. Alrred DC, Clark GM, Molina R, et al. Overexpression of HER2/neu and its relationship with other prognostic factors change during the progression of in situ to invasive breast cancer. Human Pathol 1992; 23: 974-979.

9. Rudas M, Neumayer R, Gnant MFX, et al. p53 protein expression, cell proliferation and steroid hormone receptors in ductal and lobular ins situ carcinomas of the breast. Eur J Cancer 1997; 33: 39-44.

10. Fisher DE, Schinitt SJ, Christian R, et al. Chest wall recurrence of ductal carcinoma in situ of the breast after mastectomy. Cancer 1993; 71: 3205-3028.

11. Fisher B, Dignam J, Wolmark N, et al. Lumpectomy and radiation therapy for the treatment of intraductal breast cancer. Findings from the National Surgical Adyuvant Breast and Bowel B-17. J Clin Oncol 1998; 16: 441-452.

12. Julien JP, Bijker N, Fentiman IS, et al. Radiotherapy in breast conserving treatment for ductal carcinoma in situ: First results of the EORTC randomised phase III trial 10853. EORTC Breast Cancer Cooperative Group and EORTC Radiotherapy Group. Lancet 2000; 355: 528533.

13. Mokbel K. Towards optimal management of ductal carcinoma in situ of the breast. Eur J Surg Oncol 2003; 29 (2): 191-197.

14. Fisher B, Dignam J, Wolmark N, et al. Tamoxifen in treatment of intraductal breast cancer: National Surgical Adyuvant Breast and Bowel Project B-24 randomised controlled trial. Lancet 1999; 353: 1993-2000.
15. Houghton J, George WD, Cuzick J, et al.; UK Coordinating Committee on Cancer Research (UKCCCR); Ductal Carcinoma in situ Working Party; DCIS trialists in the UK, Australia, and New Zealand. Radiotherapy abd tamoxifen in women with completely excised ductal carcinoma in situ of the breast in the UK, Australia, and New Zealand: randomised controlled trial. Lancet 2003; 362: 95-102.

16. Michaelson JS, Silverstein M, Wyatt J. Predicting the survival of patients with breast carcinoma using tumor size. Cancer 2002; 95: 713-723.

17. Silverstein MJ. The University of Southern California/Van Nuys prognostic index for ductal carcionoma in situ of the breast. Am J Surg 2003; 186: 337-343.

18. Silverstein MJ. An argument against routine use of radiotherapy for ductal carcionma in situ. Oncology (Huntingt) 2004; 17: 1511-1533.

19. Douglas-Jones, AG, Logan J, Morgan JM, et al. Effect of margins of excision on recurrence after local excision of ductal carcinoma in situ of the breast. J Clin Pathol 2002; 55: 581-586.

20. Silverstein MJ, Buchanan C. Ductal carcinoma in situ: USC/Van Nuys Prognostic Index and the impact of margin status. Breast 2003; 12: 457-471.

21. Bijker N, Peterse JL, Duchateau L, et al. Risk factors for recurrence and metastasis after breast conserving therapy for ductal carcinoma in situ: Analysis of European Organization for Research and Treatment of Cancer Trial 10853. J Clin Oncol 2001; 19: 2263-2271.

22. Solin LJ, Fourquet A,Vincini FA, et al. Mammographically detected ductal carcinoma in situ of the breast treated with breast-conserving surgery and definitive breast irradiation: long term outcome and prognostic significance of patient age and margin status. Int J Radiat Oncol Biol Phys 2001; 50: 991-1002.

23. Leonard GD, Swain. Ductal carcinoma in situ, complexities and challenges. J Natl Cancer Inst 2004; 96 : 906-920.

24. Veronesi U, Marubini E, Mariani L, et al. Radioterapia tras la cirugía conservadora de la mama en el carcinoma mamario de tamaño reducido: resultados a largo plazo de un ensayo de distribución aleatoria. Ann Oncol (Edición Española) 2001; 10: 1250-1257.

25. Vicini FA., Remouchamps V., Wallace M. et al. Ongoing clinical experience utilizing 3D conformal external beam radiotherapy to deliver partial breast irradiation in patients with early-stage breast cancer treated with breast-conserving therapy. Int. J. Radiat Oncol Biol. Phys 2003; 57: 1247-1253

26. Formenti SC., Truong MT., Goldberg JD, et al. Prone accelerated partial breast irradiation after breast conserving surgery: Preliminary clinical results and dose- volume histogram analysis. Int. J. Radiat Oncol Biol. Phys 2004; 60: 493-504.

27. Ott OJ., Potter R., Hammer J, et al. Accelerated partial breast irradiation with Iridium-192 multicatheter PDR/HDR brachytherapy. Strahlenther Onkol 2004; 180: 642-649. 
28. Polgar C., Major T., Fodor J, et al. High-dose-rate brachytherapy alone versus whole breast radiotherapy with or without tumor bed boost after breast-conserving surgery: Seven-year results of a comparative study. Int. J. Radiat Oncol Biol. Phys 2004; 60: 1173-1181.

29. Gatzemeier W, Orecchia R, Gatti G, et al. Intraoperative strahlentherapie (IORT) in der behandlung des mammakarzinoms -eine neue therapeutische alternative im rahmen der brusterhaltenden therapie? Strahlenther Onkol 2001; 177: 330-337.

30. Early Breast Cancer Trialists' Collaborative Group. Effects of radiotherapy and surgery in early breast cancer: an overview of the randomized trials. N Engl J Med 1995, 333: 1444-55.

31. Burstein HJ, Polyak K, Wong JS, et al. Ductal carcinoma in situ of the breast. N Engl J Med 2004; 350:1430-1441.

32. Perez-Calatayud J, Petschen I, Carmona V, et al. Radioterapia en el tratamiento conservador del cáncer de mama. Rev Oncol, 2002, Supl 2: 44-50.

33. Tubiana M. Repopulation in human tumors, a biological background for fractionation in radiotherapy. Acta Oncol 1988; 27: 83-88.

34. Fowler J, Lindstrom M. Loss of local control in radiotherapy. Int J Radiat Oncol Biol Phys 1992; 23: 457-467.

35. Huang J, Barbera L, Brouwers M, et al. Does delay in starting treatment affect the outcomes of radiotherapy? A systematic review. J Clin Oncol 2003; 21: 555-563.

36. Herbert-Croteau N, Freeman C, Latreille J, et al. Delay in adyuvant radiation treatment and outcomes of breast cancer review. Breast Cancer Res Treat 2003; 74: 77-94.

37. Herbert-Croteau N, Freeman C, Latreille J, et al. Delay of radiation therapy and outcomes of breast cancer. J Clin Oncol 2004; 22: 1342-1343.

38. Recht A. Impact on Outcome of delay in starting radiotherapy. J Clin Oncol 2004; 22: 1341-1342.

39. Canadian consensus document. Clinical practice guidelines for the care and treatment of breast cance . Can Med J Assoc 1998; 3: 158.

40. Barrett-Lee P. Reducing delays in cancer treatments: some targets. A report of the Joint Council for Clinical Oncology, Royal College of Physicians and Royal college of Radiologists, 1993.

41. Benk V, Joseph L, Fortin P, et al. Effect of Delay in initiating radiotherapy for patients with early stage breast cancer. Clinical Oncology 2004; 16: 6-11.

42. Herbert-Croteau N, Freeman C, Latreille J, et al. A population-based study of the impact of delaying radiotherapy after conservative surgery for breast cancer.. Breast Cancer Res Treat 2004; 88: 187-196.

43. Robertson JM, Clarke DH, Pevzner MM, et al. Breast conservation therapy: severe breast fibrosis after radiation therapy in patients with collagen vascular disease. Cancer 1991; 68: 502-508.

44. Chen AM, Obedian E, Haffty BG, et al. Breast conserving therapy in the setting of collagen vascular disease. Cancer J 2001; 7: 480-491.
Correspondencia:

Dr. F. J. Andreu Martínez

Servicio de Oncología Radioterápica

Hospital Universitari Sant Joan

Crta. Ncal. 332 Alicante-Valencia, s/n

E-03550 Sant Joan d'Alacant (Alicante)

andreu_fra@gva.es. 